but an introduction that explained the most important changes in our understanding in the past twenty years or so would have been a great help to readers.

All in all this first volume of The Shorter Science and Civilisation in China provides a splendid orientation to the cultural matrix of science and technology. It will motivate many

\section{Electrical breakdown phenomena in gases}

Electrical Breakdown of Gases. Edited by J. M. Meek and J. D. Graggs. Pp. 878. (Wiley: Chichester, UK, and New York, 1978.) £35; \$75

THE book of the same title published in 1953 is well known as a standard reference book to engineers and physicists concerned with the many aspects of electrical breakdown phenomena in gases. This welcome new version, edited by the original authors, updates much of the material in the original volume and takes account of the many advances made over the past 25 years. Inevitably the contents reflect changes of emphasis and new areas of current interest. Discussion of lightning discharges and glow-to-arc transitions is now omitted but a chapter on laser induced breakdown has been included.

As in the original volume, chapter 1 is concerned with a discussion of the fundamental collision processes relevant to breakdown phenomena. This includes basic definitions and a description of electron scattering processes, negative ion formation, collisions involving molecular species, electronion and ion--ion recombination, and drift and diffusion of electrons and ions.

Chapter 2 is concerned with vacuum breakdown including a discussion of field emission, microdischarges and microparticle pre-breakdown phenomena. The discussion of spark breakdown in uniform fields in chapter 3 includes a full description of prebreakdown ionisation in gases in steady-state conditions, electron avalanches, ionisation coefficients, and temporal growth of ionisation in impulse conditions.

A general treatment of corona discharges is given in chapter 4, and chapter 5 is concerned with spark breakdown in non-uniform fields. Breakdown voltage characteristics in both uniform and non-uniform fields are considered in chapter 6 for a wide range of gases and gas mixtures. readers to seek out more recent discoveries and interpretations. A large public will await Ronan's coming adaptations of Needham's histories of science, techniques and medicine.

N. Sivin is Professor of Chinese Culture and of the History of Science at the University of Pennsylvania.

Chapter 7 deals with the time lag between the application of an impulse voltage and the consequent breakdown of a gas in terms of the so-called 'statistical time lag' and the formative time lags associated with the initiation and development of a discharge across a gap. In chapter 8 , high frequency breakdown of gases is discussed in terms of radio and ultra high frequencies in air and sulphur hexafluoride.

Laser induced electrical breakdown of gases is discussed in chapter 9. The intensity of a focused monochromatic beam is considered, together with its application to multiphoton

\section{Liquid properties}

Liquids and Their Properties: A Molecular and Macroscopic Treatise with Applications. By H. N. V. Temperley and D. H. Trevena. Pp.274. (Wiley: Chichester, UK and New York, 1978.) $£ 15$.

THE research work of the two authors of this book, Professor Temperley and Dr Trevena, lies, respectively, in the statistical mechanics of phase transitions and in the experimental study of the physical properties of liquids. These fields exemplify the 'molecular' and the 'macroscopic' of the book's subtitle.

In the first field we have chapters 1-4 on intermolecular forces and statistical mechanics, chapter 10 on liquid structure and chapter 12 on transport properties. The rest of the book is mainly macroscopic: changes of phase, hydrodynamics and acoustics, liquids under pressure and tension (an interest shared by the authors), the gas-liquid surface, mixtures and solutions, and non-newtonian liquids. There is a final chapter on the unusual properties of liquid helium.

To cover this range of material in 270 pages allows only an elementary treatment. The book aims to provide "what every good scientist, whether in research, teaching, industry or medicine should know about liquids." It is, perhaps, most successful in doing this on absorption, ionisation and breakdown. Spark channels, which in recent years have become important in the design of opto-electronic devices of high sensitivity are considered in chapter 10. A short description of electrode effects is given in chapter 11 .

Nine different well established researchers have contributed to this volume and they and the editors should be congratulated in achieving an unusual uniformity of style for a book of this type. Unnecessary overlap of material between chapters is minimal and the many excellent diagrams and illustrations throughout the book add to its attractiveness. It is a very readable book and should appeal to specialist researchers, postgraduate students, and readers whose interests lie only on the periphery of the general field of breakdown phenomena.

H. B. Gilbody

H. B. Gilbody is Professor and Head of the Department of Pure and Applied Physics at The Queen's University of Belfast, Northern Ireland.

the macroscopic side, where the more descriptive narrative places less demands on the reader. The classical equations of hydrodynamics are derived painlessly with scarcely a result in vector notation that is not followed by its cartesian equivalent. Statistical mechanics is less easily handled this way and its equations have to be stated rather than derived if the book is to cover the wide range of subjects promised by the chapter titles.

Within these limitations the authors succeed in giving convincing and easily read accounts of their chosen topics. They are not entirely successful in the admittedly difficult job of relating the molecular side to the macroscopic. Moreover, some of the molecular theories discussed are now quite obsolete, such as the 'hole' and 'tunnel' theories of liquids and the lattice theories of liquid mixtures. There is a place in books of this kind for theories that are technically obsolete if they are physically informative (the authors rightly describe the theories of van der Waals), but not for theories which are now obsolete and which never did do justice to the basic physics. More recent and useful theories are, however, also described clearly, with particular emphasis on those put forward by the late Professor J. G. Kirkwood, to whom the authors rightly pay tribute.

The book is well produced and unusually free from misprints.

\section{J. S. Rowlinson}

J. S. Rowlinson is Professor of Physical Chemistry in the University of Oxford, UK. 